\title{
Global Forum of Cancer Surgeons: Position Statement to Promote Cancer Surgery Globally
}

Chandrakanth Are, MD, MBA ${ }^{1}$, David L. Bartlett, $\mathrm{MD}^{1}$, Aviram Nissan, $\mathrm{MD}^{2}$, Zippel Dov, $\mathrm{MD}^{2}$, Arnab Gupta, $\mathrm{MD}^{3}$, Dhairyasheel Savant, $\mathrm{MD}^{3}$, Juan Enrique Bargallo-Rocha, $\mathrm{MD}^{4,5}$, Hector Martinez Said, $\mathrm{MD}^{4,5}$, Alexandre F. Oliveira, MD, $\mathrm{PhD}^{5,6}$, Héber S. de Castro Ribeiro, $\mathrm{MD}, \mathrm{PhD}^{5,6}$, Augusto Leon, $\mathrm{MD}, \mathrm{MSCCh}^{7}$, Yuko Kitagawa, $\mathrm{MD}, \mathrm{PhD}^{8}$, Kazuhiro Yoshida, $\mathrm{MD}, \mathrm{PhD}^{8}$, Han-Kwang Yang, $\mathrm{MD}, \mathrm{PhD}^{9}$, Do Joong Park, MD, PhD ${ }^{9}$, Ashraf Zaghloul, $\mathrm{MD}^{10}$, Wael A. Gawad, MD, PhD ${ }^{10}$, Andrew McKay, MD ${ }^{11}$, Lucy K. Helyer, MD ${ }^{11}$, Haroon Javaid Majid, MD, FRCS $^{12}$, Muhammad Arshad Cheema, MD, FRCS ${ }^{12}$, Gong Chen, $\mathrm{MD}^{13}$, Alessandro Gronchi, MD ${ }^{14}$, Tibor Kovacs, PhD, FRCS, FEBS ${ }^{15}$, and Domenico D'Ugo, MD, FACS $^{14,15}$

${ }^{1}$ Society of Surgical Oncology, Rosemont; ${ }^{2}$ Israeli Society of Surgical Oncology, Ramat Gan, Israel; ${ }^{3}$ Indian Association of Surgical Oncology, Miraj, Maharashtra, India; ${ }^{4}$ Sociedad Mexicana de Oncologia, Mexico City, Mexico; ${ }^{5}$ Latin American Society of Surgical Oncology, Salvador, Brazil; ${ }^{6}$ Brazilian Society of Surgical Oncology, Salvador, Brazil; ${ }^{7}$ Catholic University of Chile, Santiago, Chile; ${ }^{8}$ Japanese Society of Gastroenterological Surgery, Tokyo, Japan; ${ }^{9}$ Korean Society of Surgical Oncology, Seongnam-si, Korea; ${ }^{10}$ Egyptian Society of Surgical Oncology, Cairo, Egypt; ${ }^{11}$ Canadian Society of Surgical Oncology, Toronto, Canada; ${ }^{12}$ Surgical Oncology Society, Lahore, Pakistan; ${ }^{13}$ Chinese Society of Clinical Oncology, Nanjing, China; ${ }^{14}$ Italian Society of Surgical Oncology, Napoli, Italy; ${ }^{15}$ European Society of Surgical Oncology, Brussels, Belgium

Cancer is one of the two major leading causes of death worldwide. The updated data for 2018 from GLOBOCAN noted that the cancer burden has risen to 18.1 million new cancer cases and 9.6 million cancer-related deaths. ${ }^{1}$ It is estimated that this burden will alarmingly rise to 29.5 million new cases and 16.3 million cancer-related deaths by 2040 (Fig. 1). ${ }^{1}$ Some worrisome aspects of this rising burden include higher burden in low-to-middle income countries (LMIC's; Asia and Africa account for more than $54 \%$ of new cancer cases), higher rise in burden in women, and inequitable access to timely and safe cancer care. ${ }^{1}$

Several organizations have dedicated their efforts towards addressing the challenges of tackling this rising cancer burden. The Union for International Cancer Control (UICC) has noted this to be an urgent global challenge and

(C) Society of Surgical Oncology 2020, corrected publication 2021

First Received: 6 March 2020

C. Are, $\mathrm{MD}, \mathrm{MBA}$

e-mail: care@unmc.edu issued a clear call for action urging the governments to take measures to scale up prevention, early detection and diagnosis, treatment, and care services. ${ }^{2}$ The laudable Treatment for All initiative launched by the UICC encompasses four components: improving the quality of cancer data for public health use, increasing the number of people with access to early detection and accurate cancer diagnosis, providing timely and quality treatment for early and metastatic disease for all, and providing a basic supportive and palliative care service for all. ${ }^{3}$

While all these efforts are essential and commendable, a consistent theme through all of these initiatives is the inadequate emphasis placed on the value and the role of surgery. ${ }^{4}$ The Lancet Oncology Commission noted that, by the year 2030, nearly 45 million additional cancer procedures will be needed, although only $25 \%$ of those patients will have access to safe, timely, and quality surgical care (Fig. 1). ${ }^{4}$ More than $80 \%$ of cancers, in particular solid tumors, will at some stage benefit from surgical intervention for diagnostic, therapeutic, and palliative purposes. Many patients may require surgical intervention more than once. 


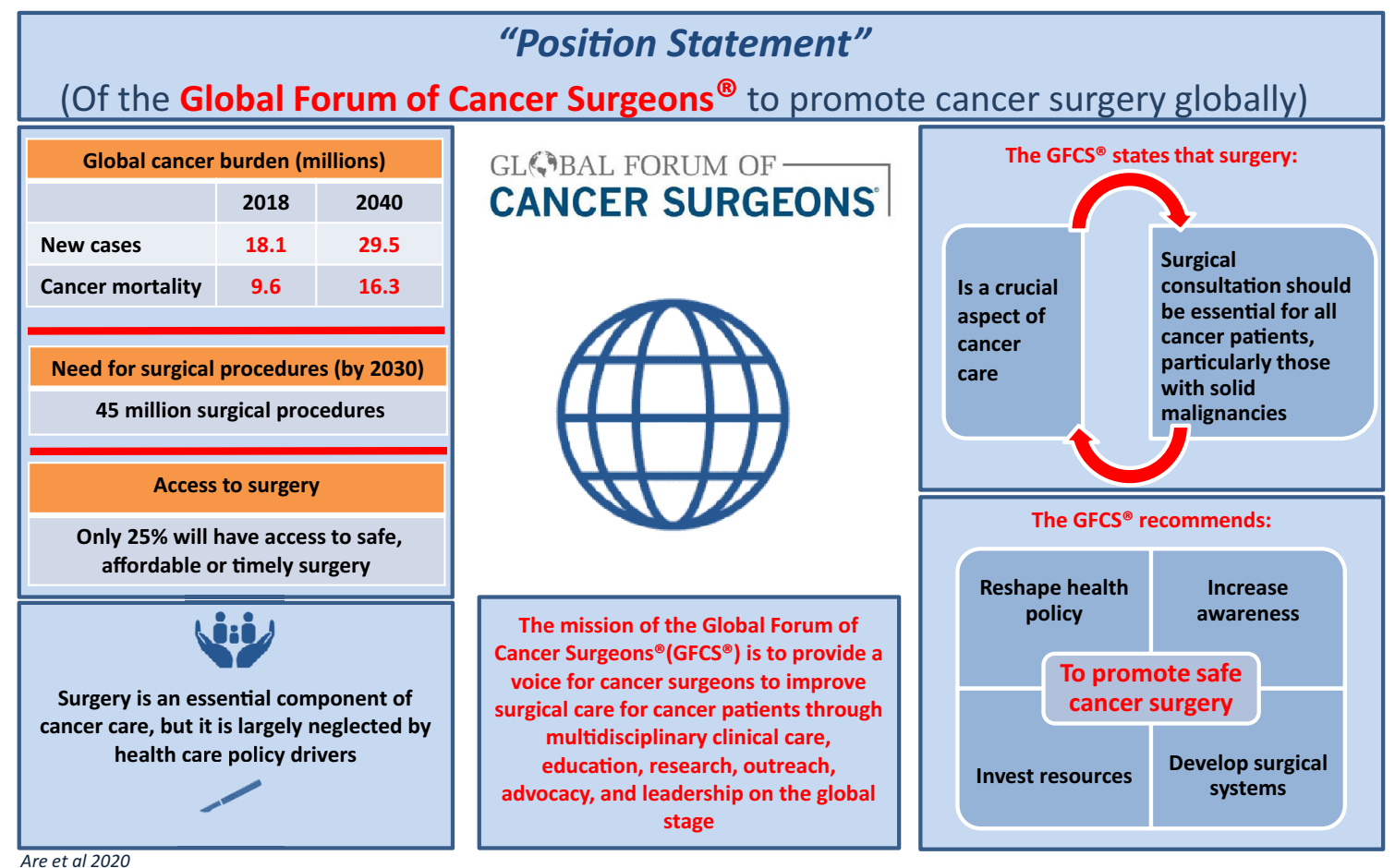

FIG. 1 Global forum of cancer surgeons: position statement to promote cancer surgery globally

Surgical care is particularly neglected in LMIC's, which often lack multidisciplinary and complementary facilities. In this context, the National Institute for Health Research (NIHR) Global Health Research Unit on Global Surgery recently stressed the need of prioritizing research and care for surgical patients in LMIC's. The domains of main concern included the outcomes of cancer surgery and perioperative care. ${ }^{5}$ However, these issues affect surgical oncology worldwide.

The evaluation of surgical outcomes (in a process of continuous improvement and standardization) can significantly impact surgical quality as well as lead to costcontrolling initiatives. Such models have been well received and implemented in a number of settingsbut need to be disseminated further. A recent study based on the American College of Surgeons' National Surgical Quality Improvement Program conducted on more than 200,000 patients who underwent major cancer-related procedures including colectomy, esophagectomy, hepatectomy, pancreatectomy, and proctectomy documented that National Cancer Institute-designated cancer centers (NCI-CCs) had better outcomes when compared with non-NCI-CCs. ${ }^{6}$

It has to be stressed that surgical quality in cancer care is not confined to reducing the odds of the adverse events (morbidity), but it should encompass more sophisticated measures, including the adherence to clinical and pathological standards of staging, the multidisciplinary setting of care, survival trends, patient-reported outcomes following the procedure, and finally quality of life.

Surgery is a very effective and highly essential component of the multidisciplinary care of the cancer patient. In the present day, surgical intervention can be accomplished with reduced mortality and morbidity. The added benefits of surgery include that this can be delivered usually in one setting with costs much lower than some of the other modalities and can deliver durable benefits including cure for some. Nonetheless, surgery for cancer care does not receive the attention it deserves. The vital role of surgery also gets crowded out by the other aspects, modalities, and fields related to cancer care. In addition, the imbalance of pressing concerns and the rising demands on resources relegates surgery to the lower tiers of the priority list for governments, elected officials, and healthcare policy drivers.

To address this imbalance between the importance and the inadequate emphasis on surgery for cancer care on the global stage, the Global Forum of Cancer Surgeons (GFCS) was formed in 2017 under the auspices of the Society of Surgical Oncology (SSO). ${ }^{7}$ The mission of the GFCS is to provide a voice for cancer surgeons to improve surgical care for cancer patients through multidisciplinary clinical care, education, research, outreach, advocacy, and leadership on the global stage. ${ }^{7}$ The GFCS continues to focus its efforts on creating a single cohesive voice for 
cancer surgeons across the world to address the barriers and highlight the importance of surgical care for cancer patients. ${ }^{8,9}$

This voice of the GFCS can be very powerful since its constituent members represent greater than $75 \%$ of the global cancer burden in terms of the new cancer cases and cancer-related mortality. At its third annual meeting conducted at SSO 2019, the members of the GFCS vigorously reiterated the need for emphasizing the role of surgery in cancer care. It was postulated to develop a position statement that highlights the importance of surgery. This position statement that reflects the opinions of surgical leaders can be very effective as it represents the majority of the cancer burden of the world. The position statement can be used by cancer surgeons and their leaders as a call for action to lobby governments, elected officials, and health policy drivers to elevate surgery to its much-needed level of prominence.

The Global Forum of Cancer Surgeons hereby states that (Fig. 1):

1. Surgical care is a crucial and vitally essential aspect of multidisciplinary cancer care.

2. Surgical intervention is the only therapeutic modality that can help cancer patients across the spectrum of care, ranging from diagnosis, treatment, to palliation.

3. Surgical intervention can offer cure to some patients with various malignancies.

4. Surgical interventions can usually be delivered on one occasion.

5. Surgical care can be tailored to be delivered across the spectrum of resource settings.

6. Surgical care can be delivered with acceptable to minimal mortality and morbidity.

7. Surgical care can be delivered with costs similar to or lower than the other treatment modalities.

8. Surgical consultation should be essential for all patients diagnosed with cancer, in particular those with solid malignancies.

To accomplish these, the Global Forum of Cancer Surgeons recommends the following actions (Fig. 1):

1. To reshape health policy at local, regional, national, and global levels that highlights the prominent role of surgery and places it at the forefront of cancer care

2. To realign the cancer advocacy efforts that includes a critical emphasis on the value of surgery and its related disciplines in the context of multidisciplinary care

3. To increase awareness of governmental agencies, nongovernmental agencies, and elected officials of the critical role of surgery in cancer care
4. To advocate for greater involvement of governmental agencies, nongovernmental agencies, and elected officials in promoting surgical care for cancer patients

5. To lobby governments and elected officials for providing and increasing resource support to achieve optimal, timely, safe, and quality-driven delivery of surgical care for cancer patients

6. To increase efforts and support to develop valuedriven and sustainable infrastructure (adequate operating room capacity and ancillary needs) to deliver safe surgical care

7. To increase efforts and resources to develop highquality and standardized educational platforms to train competent cancer surgeons

8. Through support and optimal educational platforms, to support the development of an adequate workforce of competent cancer surgeons

9. To provide support that can build novel (resourcestratified) research platforms that enable cancer surgeons to conduct specific research in the surgical aspects of cancer care and thereby deliver evidencebased care

10. To provide support for cancer surgeons to participate in professional development and continually improve their outcomes

It is anticipated that this position statement highlights the inadequacies and simultaneously reiterates the important role that surgery can play in the care of cancer patients. The constituent members of the Global Forum of Cancer Surgeons represent more than $75 \%$ of the global cancer burden by incidence and mortality, which should lend the much needed credence and importance to the position statement of this kind. We expect that this position statement can be used by any responsible entity that intends to promote the vital role of surgery as a part of the multidisciplinary care of cancer patients on the global stage.

\section{REFERENCES}

1. https://gco.iarc.fr/. Accessed Aug 2019.

2. https://www.uicc.org/news/new-global-cancer-data-globocan-201 8. Accessed Aug 2019.

3. https://www.uicc.org/what-we-do/advocacy/treatment-all. Accessed Aug 2019.

4. Sullivan R, Alatise O, Anderson BO et al. Global cancer surgery: delivering safe, affordable, and timely cancer surgery. Lancet Oncol 2015. 16: 1193-24

5. Nepogodiev D, Moore R, Biccard B et al. Prirotizing research for patients requiring surgery in low-and middle-income countries. $\mathrm{Br}$ J Surg 2019;106: e113-e120

6. Liu JB, Berian JR, Liu Y et al. Trends in perioperative outcomes of hosptials peforming major cancer surgery. J Surg Oncol 2018;118: 694-703 
7. Are C, Coit DG, McMasters KM et al. Global Forum of Cancer Surgeons: Declaration of Intent. Ann Surg Oncol. 2017;24: 2429-31

8. Are C, McMasters KM, Giuliano A et al. Global Forum of Cancer Surgeons: a steady voice for cancer surgeons to improve surgical care for cancer patients globally. Ann Surg Oncol. 2018;25: 2114-16
9. Are C, McMasters, Giuliano A et al. Global Forum of Cancer Surgeons: Perspectives on barriers to surgical care for cancer patients. Ann Surg Oncol. 2019;26: 1577-82

Publisher's Note Springer Nature remains neutral with regard to jurisdictional claims in published maps and institutional affiliations. 